\title{
La gestión de la diversidad en las empresas: desde las acciones afirmativas a las políticas de igualdad de oportunidades y las repercusiones en el contexto Español
}

\author{
Debora GotTardello \\ Investigadora en formación \\ Departamento de Gestión de empresa, Universitat Rovira i Virgili
}

DOI: $10.1387 /$ reves. 17538

Fecha de entrada: 02/04/2016

Fecha de aceptación: 06/06/2016

Sumario: Introducción. Origenes de la gestión de la diversidad. Políticas de igualdad de oportunidades. La diversidad en las empresas. Diversidad en España. a) Procesos migratorios. b) La incorporación de los inmigrantes en el mercado laboral español. Diversidad cultural e integración en el mercado laboral español actual. Conclusiones. Bibliografía.

\section{Resumen:}

Este articulo pretende visibilizar como la diversidad ha sido y sigue constituyendo históricamente un aspecto problemático al que se han enfrentado los responsables de los recursos humanos. Se analizará como España, después de la gran emigración hacia latino América y zona más prosperas de Europa del siglo XIX.XX, pasa a ser un país receptor de inmigrantes cuyas repercusiones se manifiestan en el mercado de trabajo, el cual se hace más diferenciado conllevando al incremento de la desigualdad en la vida laboral. En este contexto, la gestión de la diversidad se está convirtiendo en uno de los principales componentes de la cultura empresarial, por lo que puede llegar a afectar de forma significativa la eficiencia de la empresa y, por consiguiente, a su posición competitiva..

\section{Palabras clave:}

gestión, diversidad; políticas de igualdad de oportunidades; migraciones; España; acciones afirmativas. 


\begin{abstract}
:
This article aims to make visible how diversity has historically been and still is a problematic issue faced by those responsible for human resources. It will be analyzed how Spain, after the great migration of the twentieth century, becomes a host country for immigrants whose effects are manifested in the labor market, which is more different and has led to increased inequality in the labor market. In this context, diversity management is becoming one of the major components of the organizational culture, which may affect significantly the efficiency of the company and therefore its competitive position.
\end{abstract}

\title{
Keywords:
}

management, diversity; equal opportunities policies; migration; Spain; affirmative action.

\section{Claves Econlit:}

J150, P310, Z130

\section{Introducción}

Los acontecimientos económicos como la internacionalización de las empresas, la globalización de los mercados y los fenómenos sociales tales como la presencia femenina en el mercado laboral y la tendencia ascendente de los flujos migratorios, han infundido transformaciones sustanciales en las organizaciones. Dichas transformaciones se han concretado en una gran diversidad de género, cultura y religión en las plantillas de las empresas (Barberá, Heredia,2004) que, al estar formadas por personas de distintos países, culturas, religiones, grupos de edad y géneros, requieren nuevas formas de cooperación, para abordar los problemas relativos a la diversidad.

Uno de los principales desafíos de las organizaciones globales es fomentar una gestión de recursos humanos capaz de integrar y valorizar la diversidad, y, sin duda, desarrollar una cultura organizativa que tenga en cuenta la diversidad existente, y que sepa identificar diferencias personales, pero también puntos comunes, y adaptarlos a la cultura de la empresa, la cual también puede estar ampliamente marcada por su país de origen. La diversidad es un aspecto problemático al que se enfrentan los responsables de los recursos humanos y su gestión, conocida con el término «diversity management», nace para dar respuesta a la exigencia de los managers de gestionar eficazmente los diferentes recursos humanos disponibles.

De la solución de esta cuestión depende, entre otras cosas, el éxito de una empresa. Para las áreas de la dirección de recursos humanos, la gestión de la diversidad se está convirtiendo en uno de los principales compo- 
nentes de la cultura empresarial, por lo que puede llegar a afectar de forma significativa la eficiencia de la empresa y, por consiguiente, a su posición competitiva.

Como veremos, las políticas de gestión de la diversidad, además de un coste para las empresas, también supone una inversión de la que la empresa intentará obtener el máximo rendimiento o el máximo retorno posible, en cuanto la fuerza de trabajo gestionada correctamente puede resultar competitiva a nivel internacional.

\section{Origenes de la gestión de la diversidad}

Dentro del estudio de las Organizaciones, la diversidad es un ámbito de investigación relativamente reciente. La relación entre la diversidad y estudios organizacionales empezó a tener las primeras manifestaciones en los años '80, por la necesidad de los gerentes de las empresas de gestionar los diferentes flujos de mano de obra (Janssens y Steyaert, 2003), dictados por los cambios a nivel mundial ${ }^{1}$.

El interés por la gestión de la diversidad halló particular precedente en el derecho de los Estados Unidos y representa la última manifestación de un camino finalizado con la integración de las diversidades en las empresas que comenzó con el desarrollo de las políticas de Affirmative Action y continuado hasta mediados de los años '70 con el fomento de Programas de igualdad de oportunidades.

La acción afirmativa ( Affirmative Action) fue introducida en Estados Unidos en los ańos 60, como resultado de los movimientos por los derechos civiles, que llevaron a la firma de la Orden Ejecutiva (Executive Order 11246), contenida en la Ley de Derechos Civiles (Civil Rights Act 1964) firmada por el Presidente Lyndon B. Johnson, y constituye el primer intento de construir una nueva manera de entender las relaciones sociales, que hasta aquel entonces habían favorecido determinadas categorías de individuos, y poder así integrar la diversidad dentro de las organizaciones.

En Europa se utiliza la expresión Acción Positiva para designar lo que en Estados Unidos y en otros países anglófonos como Gran Bretaña se conoce como "acción afirmativa» (affirmative action). El origen de este término procede de una ley estadounidense de 1935 enmarcada en el ámbito del derecho laboral, pero adquirió significado especifico de política pública (policy), en el ámbito de la reacción jurídica a las protestas protagonizadas

\footnotetext{
${ }^{1}$ La globalización es el fenómeno que ha condicionado y sigue condicionando la composición social de los grupos.
} 
por la población afro-americana y otras minorías y movimientos de contestación social que de algún modo da origen asimismo al derecho anti-discriminatorio, en el que la acción positiva es fundamental (Ziliani, 2011).

La Acción Afirmativa o Positiva (Affirmative Action) fue una medida tendente a eliminar la discriminación por motivos raciales en el empleo. El núcleo de la acción era implementar programas diseñados a poner de manifiesto las condiciones de desigualdad y disparidad de tratamientos, así como fomentar políticas dirigidas a integrar y ayudar a los individuos estadounidenses en las relaciones con la sociedad en que vivían. La medida, que según las primeras disposiciones legislativas abogó por la reducción de las disparidades, a través de un aumento en el porcentaje de individuos de grupos minoritarios, constituyó el punto de ruptura en la sociedad norteamericana, formada por grupos sociales mixtos y en algunos casos heterogéneos (Cahn, 2013). Desde un punto de vista puramente histórico también, se puede afirmar que la regulación de la Ley de Derechos Civiles de Estados Unidos de 1964, en su título VII estableció la prohibición de prácticas discriminatorias en el trabajo por razones de raza, etnia, religión, género y nacionalidad, implantando una nueva manera de entender las relaciones sociales, que hasta ese momento, habían favorecido a determinadas categorías de individuos (como los hombres, a expensas de las mujeres, o los blancos en detrimento de los afro-americanos) (González, 2014)..

El término Acción Afirmativa, explica el proceso activo de la acción de lucha contra los abuso de las clases sociales. Se hablará de la acción afirmativa, para poder comprender como las políticas sociales implementadas por el Estado pueden ser utilizadas para mejorar el conocimiento organizacional sobre la consideración de la diversidad. Las acciones de carácter social implementadas por el Estado Americano, de hecho, representan un ejemplo de intervención legislativa de referencia, para poder entender las corrientes teóricas que se desarrollarán en los años siguientes a la promulgación de las medidas y que servirán de fundamento para el reconocimiento y valoración positiva de la diversidad (Lempert, 2014). Haciendo referencia a la acción afirmativa, se explica la importancia de la conciencia de la existencia de la diversidad, a partir del ámbito macro (un estado por ejemplo), y el intento de aplicarla al micro-entorno (en este caso la empresa y su entorno de referencia).

Leonard (1984) analizó el impacto de la acción afirmativa en las empresas. Mientras por un lado se evidencian resultados positivos obtenidos con la aplicación de las políticas preconizadas por la Acción Afirmativa (tales como mayores empleos para los hombres afroamericanos), también se registran aumentos reales en el nivel de los salarios, mejoras que denotan no sólo mejoras cuantitativa sino también cualitativas, con respecto al pasado. 
Las políticas sociales de los gobiernos, favoreciendo la entrada en el mundo laboral de los grupos considerados más vulnerables (incluyendo a las mujeres y los individuos pertenecientes a razas diferentes a la considerada dominante), podrían inducir a los mismos trabajadores (mujeres y minorías) a tener unas mejores percepciones dentro de las actividades de trabajo llevadas a cabo, debido a la mejor accesibilidad al empleo (Kalev, Dobbin y Kelly, 2006). Se trataría de lograr buenos niveles de reclutamiento así como unos estándares positivos de retribuciones y programas adecuados de promoción profesional, que a la luz de lo que se ha estudiado, no parece haber sido alcanzado completamente (Herring y Henderson, 2012). Esta crítica podría hacernos reflexionar sobre el hecho de que la valoración de la diversidad, podría conseguirse coercitivamente, como se ha tratado de imponer en Estados Unidos, y también investigando en las organizaciones, capturando las características más destacadas en relación con las variables demográficas, implementando las estrategias adecuadas con el fin de mejorar la explotación de los recursos humanos.

Las acciones de carácter social implementadas por el Estado Americano y la introducción de las políticas de acción afirmativa se han fundamentado principalmente en la responsabilidad moral y social de integrar las personas distintas entre ellas. Tenían el objetivo de corregir los errores cometidos en el pasado contra los afroamericanos, los latinos y de todos los que pertenecían a las minorías étnicas. El supuesto — que se mostró posteriormente erróneo- sobre el que se apoyaba la acción afirmativa, era poner fin a las prácticas discriminatoria del pasado, favoreciendo la contratación y capacitación de personal cualificados perteneciente a grupos minoritarios.

El objetivo básico era aumentar la presencia de las minorías, de las mujeres y de los discapacitados en las áreas de empleo donde, anteriormente, estas categorías eran poco representadas, como por ejemplo en los empleos estatales (Coate y Loury, 1993). La integración de las categorías sociales desfavorecidas, por un lado, y el reconocimiento de incentivos para todos los que poseen competencias y habilidades para poder desarrollar un determinado trabajo independientemente del sexo, raza, etnia, edad, educación, por otro lado, representaban un compromiso imprescindible. Un plan de acción afirmativa, en general, incluía el análisis de las normas de una organización, la comparación estadística de la fuerza de trabajo con la fuente de mano de obra disponible para poder verificar si se hacía poco uso de las minorías.

Las opiniones sobre los efectos causados por los programas de acción afirmativa son contradictorios. Por un lado, a pesar de que la intención de la acción afirmativa era asegurar igualdad de oportunidades laborales para todos, las percepciones negativas, combinadas con la mala implementación 
a nivel organizacional, se han traducido en una política social que se considera ineficaz e injusta por algunos (Holzer y Neumark, 1999).

Por el contrario, otros autores han evidenciado cómo las políticas de acción afirmativa han producido resultados positivos (como por ejemplo un aumento en el empleo de hombres afro-americanos). Algunos estudios sobre los efectos de la igualdad de oportunidades laborales y los programas de acción afirmativa sobre la estabilidad laboral de las mujeres, revelaron que las mujeres de edad comprendida entre 20 y 54 años se habían beneficiado de las políticas de acción afirmativa para obtener una mayor estabilidad en el empleo entre el 1965 y el 1980, perdiendo algunos de los beneficios adquiridos en ańos anteriores en el período posterior de 1980 a 1988 (Uri y Mixon, 1992). Aunque las políticas de acción afirmativa han demostrado ser un fracaso, en la mayoría de los casos, este enfoque ha tenido un papel fundamental en el comienzo de una etapa de cambios. La intención inicial era utilizar este enfoque como una intervención transitoria, que diera a los directivos la oportunidad de corregir los errores cometidos en el pasado, incluyendo las injusticias y las discriminaciones. Pero se ha comprendido que, una vez corregidos estos errores, la acción afirmativa por sí sola no era capaz de ayudar a las organizaciones a beneficiarse de las diversidades adquiridas.

\section{Políticas de igualdad de oportunidades}

Las políticas de acciones afirmativas decayeron a partir de los años ochenta. No obstante, estas políticas crearon una nueva conciencia basada en las Diferencias. Según Skrentny (2002): «fue a través de las acciones afirmativas como los actores políticos indagaron y establecieron oficialmente una nueva categoría de americanos: las minorías... las acciones afirmativas fueron la base de la "política de la identidad" que caracterizó al siglo xx tardío. Creó las categorías de grupo y legitimó esas políticas».

A pesar de esto, los principios fundamentales de igualdad y de libertad y las normas constitucionales anti-discriminatorias debían impedir las diferenciaciones en función de la raza y del sexo de las personas (Ezorsky, 1991). En este contexto, la igualdad de trato tenía que alcanzarse de manera que todas las personas tuvieran las mismas oportunidades conforme a sus méritos y a su esfuerzo, pero no en función de su raza, ni su sexo, ni de su pertenencia a una determinada comunidad cultural. Así, a partir de los años ochenta junto con la acción afirmativa se desarrollaron nuevas políticas para la gestión de la diversidad, como la política de igualdad de oportunidades (Herring y Henderson, 2012). Estas políticas fueron sucesivamente impuestas a las organizaciones con el objetivo de promover sistemas 
que permitieran que todos pudieran competir desde posiciones similares, y solventar situaciones de discriminación derivadas de posiciones de educación, salud o económicas no propias de los sujetos.

El concepto de igualdad de oportunidades ha sido objeto de distintas interpretaciones. Así, por ejemplo, Tawney (1961) en su obra Equality evidencia serios problemas para explicitar una cuestión tan complicada y emplea metáforas en las que hace referencia, entre otras cosas, a tronos, asnos, zanahorias, renacuajos, escaleras, arrecifes, pararrayos y abismos.

Según Tawney (1961), la igualdad de oportunidades fue inicialmente presentada en términos negativos en lugar de positivos interpretándose «más bien como la libertad de las restricciones, como la posesión del poder, pero en la realidad igualdad de oportunidades no es simplemente cuestión de igualdad legal. Su existencia depende no simplemente de la ausencia de discapacidades sino de la presencia de capacidades. Se traduce en la medida de lo posible y sólo en la medida de lo posible, en la posibilidad de que cada miembro de la comunidad, cualquiera que sea su origen, ocupación o posición social, posea de hecho iguales oportunidades de utilizar plenamente sus recursos naturales sean físicos, de carácter y de inteligencia. En la proporción en que las capacidades de algunos son constreñidas por su entorno social, mientras que las de otros son protegidas y mimadas, la igualdad de oportunidades se convierte en una graciosa ficción».

Sartori (1992) divide la igualdad de oportunidades en dos: una igualdad de acceso y una igualdad de partida. Con igualdad de acceso se hace referencia a la no discriminación en la entrada o promoción; lo que se reconoce y se recompensa es el desempeńo o la actuación reales y, como resultado, la igualdad de mérito, capacidad o talento. «Igual reconocimiento a igual mérito, se traduce en la mayoría de las ocasiones en la fórmula de la "carrera abierta al talento", en función, y únicamente en función, de la capacidad y de los méritos». La igualdad de oportunidades como acceso está estrechamente conexa a la equidad de género, a la problemática de las mujeres y al intento de crear condiciones de igualdad de oportunidades entre hombres y mujeres por parte de las políticas públicas.

Con igualdad de partida se entiende «igualdad de condiciones iniciales (para lograr la igualdad de acceso)». Esto indicaría una igualdad en las condiciones materiales y entendiéndose así la igualdad económica.

Las últimas décadas del siglo $\mathrm{xx}$ han dado mayor trascendencia a la igualdad de oportunidades, debido entre otras cosas al progresivo consenso en torno al respeto por los principios básicos del liberalismo y de la economía de mercado.

Las políticas de igualdad de oportunidades tienen como propósito asegurar la justicia e igualad absoluta entre los miembros de una organización, dejando a los sujetos la posibilidad de poder progresar y alcanzar los resul- 
tados que puedan adquirir con sus propios méritos y esfuerzos, sin importar su raza, sexo o cultura (Gibbon, 1990). Así las políticas anti-discriminatorias que surgieron en los años sesenta y setenta del pasado siglo, con el objetivo de asegurar la igualdad de oportunidades a las personas de distintas razas, generaron una nueva conciencia basada en las diferencias, integrando así las minorías en los ámbitos en los que estaban poco representadas (Alonso, 2003).

El Auge de las acciones afirmativas comenzó su declive a finales de los ' 90 , en beneficio de las políticas de igualdad de oportunidades, que se fundamentaban en la total igualdad de los miembros de las organizaciones, $y$ que a diferencia de las acciones afirmativas no se basaban en la obligación de cuotas que hicieran a las empresas contratar un determinado número de personas pertenecientes a las clases más desfavorecidas.

En un discurso público el presidente Reagan en el 1980 afirmó: «no podemos permitir que el noble concepto de igualdad de oportunidades degenere en unas guías federales o cuotas que requieren que la raza, la etnia o el sexo, en lugar de la habilidad y la cualificación, sean el principal factor de contratación o de educación» (Lahey, 2005). Las habilidades, los méritos, el esfuerzo han sido elementos fundamentales de la igualdad liberal, poniéndose en contra de las acciones afirmativas.

Las acciones afirmativas, y las medidas de discriminación inversa nacidas para declarar inconstitucionales algunas prácticas surgidas al amparo del concepto de acción positiva, han encontrado regulación jurídica internacional. Hay diferentes opiniones, por una parte hay quienes consideran, desde una perspectiva liberal, que la igualdad debe entenderse como tratar a las personas en manera proporcional a sus méritos individuales (Garrison y Modigliani, 1994). Otros opinan que existen otros criterios relevantes que se deben tener en cuenta. Piensan que las circunstancias contribuyen a los logros individuales y, por ende, deben de modificarse para que la igualdad resida en las oportunidades (Wejnert, 2014).La característica principal de la política de igualdad de oportunidades es promover la igualdad absoluta entre los miembros de la organización, sin tener en cuenta su género, etnia, religión, etc., en sustancia sin tener en cuenta su singularidad. El objetivo es recompensar y tratar a los miembros de la organización sólo sobre la base de un criterio relacionado con el trabajo. Aspectos étnicos o culturales no deben ser considerados criterios pertinentes para premiar o castigar a una persona (Liff, (1995.

A diferencia del enfoque de acción afirmativa, la Igualdad de Oportunidades son políticas que se basan en la igualdad total de los miembros de la organización (Cockburn, 1989). Esta igualdad se agrava hasta el punto de que los miembros son "privados» de su género, nacionalidad, cultura o religión con el fin de crear una organización compuesta por todos los 
miembros de la misma. La principal limitación de este enfoque es la falta de reconocimiento y la protección de los rasgos de identidad de los empleados de la empresa.

\section{Consideraciones sobre las Politicas de igualdad de oportunidades y Acción Afirmativa}

El concepto de diversidad se ha utilizado tradicionalmente en sentido estricto, centrándose en frenar las prácticas discriminatorias en base a las características personales, pero ha sido definido también en un sentido más amplio, incluyendo las implicaciones de la cultura organizacional. Esta visión de la diversidad afecta a todos los niveles de la organización, mientras que las definiciones estrictas parecen tener una orientación similar a las Acciones Afirmativas (AA) y a las Políticas de Igualdad de Oportunidades en el Empleo (EEO).

Se han llevado a cabo varias distinciones entre Igualdad de Oportunidades en el Empleo, Acción Afirmativa y la diversidad. EEO se ha definido como dar a las personas una oportunidad justa para poder prosperar dentro del contexto laboral sin discriminaciones basadas en factores tales como la edad, la raza, el sexo o la nacionalidad (Erwee y Steger, 2001). AA se refiere a Programas que requieren que las organizaciones hagan esfuerzos especiales para reclutar a las mujeres y las minorías. La gestión de la diversidad, va más allá de la comprensión de las diferencias individuales y abarca una situación en la que la cultura organizacional logra reconocer, respetar y valorar las diferencias.

La diversidad en oposición a las políticas de igualdad de oportunidades (EEO) y las Acciones afirmativas (AA) abarca a un sector más amplio de la población activa. Mientras que las EEO y AA están amparadas por la legislación, para poder garantizar una fuerza laboral realmente diversa es indispensable que los responsables de la gestión de personas emprendan estas iniciativas. Podemos considerar que aunque las políticas de Igualdad de Oportunidades y las Acciones Afirmativas han constituido unas etapas importantes para la evolución de la gestión de la diversidad, representan solamente una pequeńa parte de su existencia (Agocs y Burr, 1996)...Hay diferentes estrategias que se pueden identificar para hacer frente a la diversidad en una organización. La diversidad parece aludir al etnocentrismo (la visualización del propio grupo como mejor), los prejuicios (prejuzgando infundadamente y negativamente a los demás) y los estereotipos (atribuyendo a los individuos características negativas asumidas por un grupo), etc., más que a la discriminación (Scheepers, 2007). Mientras que la discriminación se refiere más bien a las acciones y comportamientos, la diversidad alude a 
los supuestos, sentimientos, percepciones y creencias sobre los demás. Una gestión eficaz de la diversidad se fundamenta en el reconocimiento de los puntos en común y en la conciencia de las diferencias.

Entre los intereses de las organizaciones se halla el considerar la gestión de la diversidad como un compromiso esencial en las políticas de recursos humanos (Slater, Weigand y Zwirlein, 2008), por esta motivación la dirección y los programas han variado de manera significativa entre las corporaciones, incluyendo las oportunidades de mentoring, programas de formación, las políticas de conciliación de la vida laboral y familiar, políticas favorables a las familias, y grupos de apoyo.

Kersten (2000) resume tres aspectos principales que hacen que la gestión de la diversidad se diferencie de los enfoques anteriores en concreto de las políticas de Igualdad de Oportunidades en el Empleo y las Acciones Afirmativas. En primer lugar, la gestión de la diversidad aboga por una transformación sistémica de la organización en comparación con el énfasis en el reclutamiento / selección que era característica de los métodos más antiguos. Por lo tanto, su objetivo es cambiar la cultura de la organización para convertirla en un entorno abierto, saludable y acogedor para todos los empleados con la ayuda de una auditoria de la diversidad de la cultura de la empresa y de sus trabajadores. Según el autor la diversidad es también diferente en su retórica, no constituyendo un mandato negativo, externo, sino como un esfuerzo positivo y voluntario por parte de la organización de incluir personas con diferencias. La tercera diferencia es que los esfuerzos de diversidad se justifican con los instrumentos económicos y no jurídicos.

La diversidad en las organizaciones no está legislada, sin embargo la legislación sobre la igualdad de trato y no discriminación, cuya finalidad es ofrecer a todas las personas posibilidades equitativas y justas de acceso a las oportunidades sociales y por consecuente la igualdad de acceso al trabajo, puede ejercer de elemento de presión para las organizaciones, con el objetivo de demostrar la proactividad en este ámbito(Stone y Arthurs, 2013)

Las organizaciones que quieren subsistir en el mundo globalizado y generar ventajas competitivas deben entender las diferencias y ajustar sus políticas hacia una cultura que estimule la inclusión, permitiendo así retener mejores empleados (Podsiadlowski, Gröschke, Kogler, Springer y Van der Zee, 2013). Empleados que poseen diferentes culturas pueden compartir sus conocimientos y lo aprendido en los contextos de origen en los que se han formado y esto permite generar ideas nuevas creativas e innovadoras y crear valor para las empresas, y altos niveles de productividad, sinergias y generar la unidad de la organización a través del compromiso de los colaboradores con la misión de la empresa (Yang y Konrad, 2011). Este compromiso requiere que los mismos tengan una alta calidad motivacional actuando por motivos trascendentes (Chincilla y Cruz, 2011) 
Según otros autores (Kelly y Dobbin,1998) existe una triple visión sobre EEO, AA y Gestión de la diversidad, señalando las diferencias entre ellos aunque dicen haber significantes convergencias en la práctica. Mientras la Gestión de la diversidad propugna los valores de inclusión y respeto de las diferencias, las políticas de Igualdad de Oportunidades promueven valores como el igualitarismo y la meritocracia y las AA la preocupación para remediar los errores del pasado. Podemos por lo tanto encontrar distinciones entre la gestión de la diversidad y los otros dos enfoques, esta distinción se refiere principalmente a las diferencias en la teoría, mientras que en la práctica existe una convergencia significativa entre la gestión de la diversidad, igualdad de oportunidades en el empleo y Acción Afirmativa.

\section{La diversidad en las empresas}

La diversidad no es simplemente un reflejo de las variables con las que una organización y los individuos que la componen se identifican y clasifican. El concepto de diversidad puede ser representado como las diferencias, similitudes y tensiones que se derivan, entre los elementos que pertenecen a un grupo, de una combinación de sujetos. Lo anterior hace reflexionar sobre cómo la diversidad puede y debe ser considerada. De hecho, el estudio de la diversidad y de las organizaciones que se ven afectadas, simplemente como un conjunto de elementos diferentes entre ellos, difuminaría el concepto sobre el que rige el Diversity Mangement y que abarca la unidad del concepto relacionado con la diversidad, resultado de la implicación de factores, que como veremos más adelante, serán las variables demográficas.

El termino diversidad, referido a las organizaciones, se asocia en la literatura a las diversas maneras de entender la estructura de los grupos, de las empresas, y los individuos.

Entre los autores que se han ocupado del estudio del problema, se puede afirmar que algunos han asociado el concepto de diversidad a la Acción Afirmativa analizada anteriormente (Thomas, 2001 )o a movimientos asociados al reconocimiento de los derechos humanos, investigando sobre el origen del fenómeno, y quien la ha asociado a las consecuencias que provoca como por ejemplo los resultados empresariales. La diversidad implica una multidimensión del concepto que va más allá de la simple consideración de un grupo o de un individuo diferente porque no es conforme a los estándares. Sera necesario hablar de diversidad indicando las diferencias, las similitudes, las tensiones y los conflictos que inevitablemente se producirán dentro de los grupos. La normativa implementada en Estados Unidos, que gira en torno a la acción afirmativa, podría interpretarse a la luz de lo que se ha dicho, no sólo en referencia a sujetos en particular, sino también y sobre todo como resultado 
de construcciones mentales, de los que poseen el poder dentro de la sociedad, entendiendo el poder, no como poder político o legislativo sino como capacidad de influir y dominar los comportamientos humanos.

Para entender mejor esta opinión, serían suficientes cruzar los conceptos de diversidad y de Acción Afirmativa. Parece evidente, que la diversidad por un lado y los principios expresados por la Affirmative Acción por otro (al menos en sus primeras etapas de vida) no han considerado el papel desempeñado por las ideas dominantes, por los juicios sociales que desprestigiaban a los individuos pertenecientes a determinadas categorías sociales(Herring y Henderson, 2012). La diversidad debería ser interpretada no limitándose a considerar a los sujetos diferentes porque son minoría, sino comprender las motivaciones por las que ellos se encuentran en situación de minoría, identificar las causas y verificar las consecuencias que ellas ha generado (Ortlieb y Sieben, 2013). Solamente después de haber comprendido estos pasajes, se puede afirmar que los individuos o los grupos son potencialmente diferentes entre ellos.

En los años siguientes a la entrada en vigor del Civil Right Act, se han activado un conjunto de programas, como el del 1972, dirigidos a la implementación de programas de reclutamiento para grupos minoritarios y mujeres con el objetivo de aumentar su presencia en los empleos públicos (Kravitz, 2008). Tras la promulgación del Civil Right Act, las instituciones empezaron a entender que para poder eliminar cualquier forma de racismo eran necesarias acciones coercitivas para amparar a las clases más desfavorecidas.

Los programas implementados en los años siguientes y relativos a otras medidas normativas han evidenciado otros problemas como la discriminación inversa (Rutherglen, 2014), entendida como exclusión o limitación de la posibilidad de participación en los empleos para los individuos no incluidos en las clases sociales protegidas. Según Thomas (2001) en su obra Affirmative Action. From the perspective of diversity, es imprescindible individualizar la manera de crear un ambiente de trabajo que funcione para todos.

La Acción Afirmativa como vimos anteriormente, no tuvo los efectos deseados ya que las personas contratadas fueron marginadas, porque se consideraba que ocupaban el lugar de trabajo no por el mérito o la competencia, sino por pertenecer a grupos desfavorecidos y su trabajo no era apreciado y valorado.

A mediados de los años 70 el gobierno promovió programas destinados a eliminar la discriminación, incentivando la paridad y la igualdad, sin llegar a proteger la diversidad y por lo tanto la singularidad de los individuos (Liff, 1995).

Fue a partir de los años 80 , cuando se empezó a valorar la diversidad a través de un enfoque ético en la gestión de la diversidad en el puesto de tra- 
bajo, aceptando los seres diferentes entre sí. Para poder implementar la diversidad es fundamental poseer o crear una cultura organizacional estratégica que las empresas deciden aplicar voluntariamente permitiendo no sólo la protección y la integración de la diversidad, sino además, su desarrollo y su utilización para el logro de los objetivos empresariales.

El objetivo es promover la formación de grupos de trabajo que contribuyan al logro de los objetivos estratégicos. Esto se explica a través de la creación y la implementación de un programa organizacional destinado a crear una mayor integración de todos los individuos (Gilbert, Stead, Ivancevich, 1999) una forma de incrementar el negocio y ofrecer ventajas competitivas en diferentes maneras (Charles, 2003). Como explica Thomas (1992), la integración (basada en la valoración de diferencias), en oposición a la asimilación (resultante del cumplimiento de la organización), se convertirá en una codiciada estrategia organizacional por las siguientes razones: (1) los empleados están menos dispuestos a integrarse en la cultura organizacional dominante y (2) algunos factores pueden estar por encima de la integración. El autor sugiere, además, que la integración de diversos empleados puede ser disfuncional, con el resultado de la incapacidad para atraer y retener a personas cualificadas.

Con el fin de poner en práctica una política de gestión de la diversidad exitosa y beneficiarse de la diversidad en el trabajo, la Comisión Europea ofrece apoyo a las organizaciones intermediarias, como las organizaciones profesionales y empresariales, organismos gubernamentales y otros organismos, así como las escuelas de negocios que ofrecen consejos sobre cómo manejar la diversidad. Además, los estudios de caso sobre empresas que ya han implementado la gestión de la diversidad, deberían animar a otras empresas a tomar estas medidas (Comisión Europea, 2008). La imitación de las prácticas de gestión de la diversidad de los que las adoptaron con éxito, así como la difusión de la gestión de la diversidad a través de los profesionales y sus redes, según lo propuesto por la UE, podría dar lugar a un aumento de la homogeneidad entre empresas.

\section{Diversidad en Espańa}

Entre las características distintivas de España se evidencia la influencia directa de sus períodos históricos sobre la forma en que se entiende y gestionan las diferencias (Zapata-Barrero, 2010).

España ha sido a menudo descrita como un país crecientemente receptor de población extranjera y que podemos fechar en los comienzos del siglo xxi. Las peculiaridades propias de España como país receptor de inmigración requieren una matización acerca de su evolución histórica, puesto 
que podríamos decir que se considera un país relativamente joven en el estudio y abordaje de los movimientos migratorios (Sanz Lafuente, 2015). Para ello, es necesario atender a las diferencias que se han registrado en las últimas décadas.

Antes de ello pero parece necesario delinear algunas circunstancias históricas que ayuden a entender mejor el estado de la cuestión: a) los procesos migratorios españoles, b) la incorporación de los inmigrantes en el mercado laboral español.

\section{a) Procesos migratorios}

Entre la mitad del siglo xIx y xxi emigraron de España más de cinco millones de españoles hacia el continente americano. Los principales países de destino fueron Argentina (1,5 millones de inmigrantes) Uruguay, Brasil y Cuba. Previamente a este periodo entre el 1720 y 1770, la Corona española impulsó el éxodo desde Canarias. La mayoría del contingente estaba compuesta por familias cuyo destino fueron Cuba, Uruguay y Venezuela (Hernandez, (1988:166). Los gobiernos de latinoamerica aprobaron leyes para fomentar la inmigración considerándola como una oportunidad para su futuro en cuanto les permitía conseguir una estabilidad económica y demográfica (Palazon, 1993)

Entre el 1850-80 hubo un receso de las salidas pero, a partir de los ' 80 de este siglo, la emigración registró un incremento del 18 por ciento con destino Cuba, Venezuela y Argelia. La emigración Canaria hacia a Cuba se realizaba a través de la contrata, forma de esclavitud temporal, que obligaba a trabajar un número determinados de años en las plantaciones. Cubra tra la abolición de la esclavitud entre el siglo XIX al xx y el incremento de demanda de mano de obra se convirtó en un país receptor de inmigración en masa. La población inmigrante y la color empezaron a ser incorporadas en la sociedad y a tener los mismos derechos y el termino «raza» cobró un lugar central en las relaciones laborales desencadenando acciones de propaganda de los gobernantes (Naranjo, Santamaria Garcia, 2000). La presencia de los españoles en el mercado de laboral cubano se centraba principalmente al sector primario, la mayoría de los hombres entraban a trabajar en las plantaciones de azogar mientras que las mujeres en el tabaco, Alvarez (1995), subsistiendo diferencias elevadas de renta entre la población local y la inmigrante, Bernet (1994) constituyendo esto motivo de conflictos entre ambas(Naranjo, Santamaria Garcia, 2000).

La emigración española hacia Europa se produjo durante el siglo xx, el destino principal fue Francia, debido sobre todo al incremento del coste de la vida que durante la Primera Guerra Mundial que afectó a España 
(Nadal, 1975). Entre el 1900 y 1930 salieron 144.000 personas hacia Europa. Con la llegada de la guerra civil el proceso tuvo una paralización debido a la restricción, por parte del gobierno, de conceder pasaportes. Durante esta época la presencia de extranjeros en Espańa era reducida: se podían calcular de manera oficial 40.000 en el 1941 y 56.000 en el 1950, de los cuales solo 11.000 disponían de carta de trabajo (Cardelus y Pascual, 1979: 302).

Durante la guerra civil española se decretó la prohibición de emigrar con el objetivo de recuperar las perdidas demográficas causadas por el conflicto. Sucesivamente, en los años 50, el gobierno creó el Instituto Español de Emigración cuyo fin era asistir a los emigrantes en los procesos de búsqueda de empleo y mantenimiento del núcleo familiar (Hernandez, (1988). En 1962 tras la promulgación de la Ley de Emigración, los éxodos del país se convirtieron en un derecho tutelado por el Estado, que desarrolló mecanismo para poder amparar los emigrantes y garantizar las ayudas en el exterior.

Durante los años '50-'75 los españoles emigraron hacia las zonas más industrializadas del país, Cataluña, Madrid, País Vasco y Comunidad Valenciana, y al mismo tiempo también la emigración exterior continúo de forma destacada, sobre todo hacia Suiza, Alemania y Francia. Según el Instituto Español de Emigración, durante todo el periodo salieron dos millones de españoles (800.000 emigrantes) es decir alrededor del 7\% de la población (Osuna, (1985:39).

Durante los ańos 70 el flujo migratorio hacia Europa sufre un descenso, registrando un regreso de emigrantes españoles (entre 1975 y 1990 se registró el retorno de medio millón de emigrantes). A partir de los años 80, España pasa de ser un país de emigración a país de inmigración. No obstante, según Fambuena (1996) «es preciso puntualizar que incluso en 1994, aún había más españoles residiendo en el extranjero, que extranjeros residiendo en nuestro país, concretamente 451.364 personas».

\section{b) La incorporación de los inmigrantes en el mercado laboral español}

España ha sido desde siempre un país en el que los movimientos migratorios han tenido una importante presencia y, a lo largo de los años ' 80 , pasa a ser país de inmigración llegando a tener unos 150.000 inmigrantes. Además, estas elevadas cifras se consolidan con incrementos anuales espectaculares, especialmente durante la década 2000-2010 (Gozálvez Pérez, 2010). Desde el 2011-2013 Espańa registró un saldo migratorio negativo de 256.849 personas, debido al descenso del 4,3\% de la inmigración y un aumento del $22,7 \%$ de la emigración. 
En enero de 2003, el número de extranjeros empadronados en España supone el 6,2\% de la población total residente. Ahora España es uno de los países europeos occidentales con mayor presencia de extranjeros, se considera que el $10,1 \%$ de la población empadronada es extranjera. La población extranjera residente en España a 1 de enero de 2015 alcanza los 4,7 millones de habitantes (INE, 2015). La incorporación masiva de los inmigrantes al mercado laboral en tan escaso tiempo, la heterogeneidad en términos de nacionalidades y el aumento de la diversidad cultural y social, a pesar de ser vistos como una tendencia positiva, han supuesto un importante desafío para las organizaciones españolas. Por ejemplo, el mercado de trabajo se ha vuelto más diferenciado y la desigualdad en la vida laboral se ha incrementado. Los extranjeros que viven en España se enfrentan a desafíos importantes en la carrera profesional, a pesar de los planes oficiales para promover el empleo de los extranjeros y los enfoques positivos hacia la diversidad de las organizaciones españolas.

El hallazgo más destacado es la desventaja salarial para los inmigrantes, la cual parece reducirse con la duración de su estancia en España (conforme mejora su adaptación lingüística, laboral, y cultural), si bien con variaciones entre países y períodos. Esta diferenciación en los cargos podría considerarse el resultado de procesos discriminatorios tendentes a limitar el acceso de los inmigrantes a cierto tipo de empleos. Se considera que en España los inmigrantes ocupan puestos de trabajo cuyos salarios son más bajos y con peores condiciones laborales que los nativos y esto se debería a la adecuación a los puestos de empleo más degradados, que suelen experimentar inicialmente los inmigrantes, si bien, para algunos, esta situación tiende a mejorar, con el paso de los años.

Según algunos estudios (Ramos, Sanromá, Simón, 2013)en España, los inmigrantes ocupan puestos de trabajo de niveles inferiores, debido a la fuerte presencia de los mismos en ocupaciones no cualificadas, que requieren mayor productividad, y el estatus de los inmigrantes en España tiende a ser, por lo general, inferior respecto a otros países.

En el ámbito de la inclusión laboral de los inmigrantes se han identificado diferentes dificultades para estas minorías entre las cuales destacan la falta de correspondencia entre los niveles de estudio que algunos inmigrantes poseen y el trabajo que desempeñan, la segmentación étnica del mercado laboral ( nichos laborales para determinados inmigrantes) discriminación de género para determinadas ocupaciones, y oposiciones de incorporación laboral más reducidas para determinadas minorías culturales.

Se observan varios casos de personas con estudios medios y superiores, que, en el país de origen han desarrollado su profesión, pero, una vez en España, «renuncian» a intentar ejercerla, por diferentes razones, a pesar de poseer la cualificación necesaria para acceder a otro tipo de empleo con 
mejores condiciones y mejor consideración social, teniendo repercusiones en la integración. Esto, sin embargo, no ocurre para todos los inmigrantes.

La discrepancia salarial entre los nativos e inmigrantes depende, según algunos estudios (Ramos, Sanromá, Simón, 2013), de la región de origen de los inmigrantes: los inmigrantes procedentes de países desarrollados tendrían salarios promedio superiores y una distribución salarial más dispersa que la de los nativos, mientras que el salario de los inmigrantes procedentes de países en desarrollo sería más bajo.

No obstante podemos observar, cada vez más, una mayor integración en el empleo de los inmigrantes, los cuales van consiguiendo una mejor ubicación en el mercado de trabajo.

\section{Diversidad cultural e integración en el mercado laboral español actual}

$\mathrm{El}$ acceso al mercado laboral es un elemento fundamental en la integración social (Salazar, 1974). A través del empleo las minorías tienen ingresos y adquieren una serie de derechos y prestaciones y evitan ser marginadas.

La no discriminación es esencial de cara a la integración laboral de las personas con diferencias culturales, y les permite gozar de una igualdad de oportunidades sustancial, no meramente formal y se considera un eje necesario a la hora de analizar la integración social de las mismas.

Tras analizar los datos sobre inmigración, podemos deducir que las organizaciones españolas tienen un evidente interés en promover políticas de integración de las diversidades culturales y reducir o eliminar la discriminación. La inmigración comporta retos de diversa índole a la sociedad de acogida. No hay que olvidar que el proceso migratorio que se ha registrado en España, ha generado un valor superior para la sociedad, sobre todo teniendo en cuenta que la población inmigrante y culturalmente diferente, se ha convertido en no pocas ocasiones, en un elemento importante para el mayor progreso económico.

Los directivos para poder actuar, deberán conocer las políticas de diversidad, ser conscientes de la gestión de la misma, y tener una visión a más largo plazo, considerando la diversidad de sus miembros como un factor de enriquecimiento, debido a la presencia de "pensamientos diferentes» que aportan nuevos conocimientos, o nuevas ideas y convertir la gestión de la diversidad en una práctica en continua transformación capaz de adecuarse a la evolución de los equipos de trabajo(Fine, (1996).

Por ello, podríamos argumentar que la gestión de los recursos humanos debe ser enfocada hacia, lo que denominamos, diversidad de capital humano, en cuanto si no se gestiona adecuadamente, los grupos pueden generar unos efectos negativos que dan lugar a equipos desintegrados. 
Así, la manera en la que los empleados sean gestionados no debe depender de su género, edad, cultura de origen etc., sino de los conocimientos y capacidades que aportan a la empresa y que pueden añadir valor a la misma.

En este contexto, la gestión de la diversidad, como una iniciativa de las organizaciones, que aborde las cuestiones de la diferencia e inclusión, ha surgido como un tema importante y de actualidad (Ronda-Pérez, Agudelo-Suárez, López-Jacob, García y Benavides, 2014), primero con la aprobación de la Ley Orgánica 4/2000, de 11 de enero, sobre derechos y libertades de los extranjeros en España y su integración social, (Ley Orgánica 4/2000, de 11 de enero, art. 23) que ha hecho un esfuerzo importantes en materia de integración social, calificando como discriminatorio todo acto que «directa o indirectamente, conlleve una distinción, exclusión, restricción o preferencia contra un extranjero basada en la raza, el color, la ascendencia o el origen nacional o étnico, las convicciones y prácticas religiosas, y que tenga como fin o efecto destruir o limitar el reconocimiento o el ejercicio, en condiciones de igualdad, de los derechos humanos y de las libertades fundamentales en el campo político, económico, social o cultural.

Sucesivamente el Ministerio de Trabajo e Inmigración en el año 20102011 ha creado el proyecto GESDI sobre la «gestión de la diversidad», y en el año 2013 ha inaugurado el proyecto GESDIMEP, sobre Gestión de la Diversidad en la Pequeńa y Mediana Empresa. Ambos proyectos tienen el objetivo de mejorar la igualdad de trato y gestión de la diversidad en el mercado laboral, reforzando la integración de los inmigrantes y minorías étnicas en el ámbito de las empresas y entornos profesionales.

Según estos proyectos algunas empresas españolas han conseguido articular una "política de gestión de la diversidad cultural», que le ha permito internacionalizarse y generar mayor productividad, cambiando y enriquecemos sus culturas organizativas, para poder gestionar la diversidad cultural de una manera adecuada.

La gestión de diversidad en Espańa se ha convertido en una tematica debatida por parte de los estudiosos en el campo de los recursos humanos. Este gran interés surge de la necesidad de conocer los efectos de la diversidad en las organizaciones, para así poder evaluar el alcance y encontrar un remedio, en caso de efectos negativos. Indudablemente la diversidad, para poder generar efectos positivos dentro de una empresa, debe ser ajustada y adaptada al contexto en el que se refiere.

En primer lugar hay que decir que el impacto de la diversidad en el rendimiento del negocio, y de los individuos, no es del todo claro. Algunos estudiosos (Burger y Bass, 1979; Child, 1981) consideran que aunque las organizaciones alrededor del mundo son cada vez más parecidas a nivel macro-económico, es decir en la estructura y la tecnología utilizadas en to- 
das las culturas, a nivel micro-económico el comportamiento de las personas dentro de estas organizaciones mantiene su especificidad cultural, incluso dentro de la misma organización.

Hofstede (1980)en contraste con la mayoría de los estudios organizacionales anteriores, que incluían un número limitado de países o analizaban diferentes compañías en distintos países, encuestó a más de 116000 empleados de 40 países, que trabajaban para una sola corporación multinacional y, a raíz de su estudio, encontró diferencias significativas en el comportamiento de los empleados provenientes de diferentes países, trabajando en la misma organización, confirmando que la cultura nacional tiene gran impacto sobre los valores y actitudes en el trabajo de los empleados como vimos anteriormente. Otros estudios han demostrado que el sistema de valores de cada individuo, derivado de su cultura nacional, afecta a su manera de comportarse en las organizaciones, la manera de interactuar entre ellos y con los demás miembros de diferentes culturas, y sus conceptos de organización, gestión, toma de decisiones, liderazgo, planificación y gestión de conflictos ((Burger y Bass, 1979; Hofstede, 1980). La diversidad tiene diferentes consecuencias, por ejemplo, en los niveles salariales y en la promoción profesional entre hombres y mujeres (Pfeffer, 1981), o más generalmente la diversidad en términos de edad, la cultura o el origen étnico, puede dar lugar a malentendidos. Esto conlleva que la diversidad provoca muchos efectos sobre el entorno, las personas, grupos y empresas, por lo que concebir las organizaciones como entidades sociales complejas incluiría este tipo de concepto (Bassett-Jones, 2005).

Podemos considerar que tambien en España, con una población cada vez más diversa dentro de las organizaciones, la comunicación intercultural efectiva se convierte en la máxima prioridad en los entornos de trabajo diversos; por lo tanto, la diversidad cultural puede ser ventajosa o perjudicial para las organizaciones en función de la naturaleza y estructura de la comunicación de los miembros de las organizaciones.

\section{Conclusiones}

La gestión de la diversidad cultural, étnica, de genero etc. y la igualdad de oportunidades en el acceso al empleo, está tomando conciencia en España, la cual se está empezando a adaptarse a la realidad de una sociedad que ha cambiado drásticamente en tamańo y en composición en muy poco tiempo.

Por todo ello, las políticas activas de diversidad deben apoyar los procesos de convivencia e integración, en tanto en cuanto estamos ante fenómenos que requieren un adecuado y justo tratamiento. La gestión de la 
diversidad debe ayudar a conseguir el mutuo respeto de creencias, culturas y concepciones, sobre la base de normas claras y adecuadas que garanticen la seguridad e integración de las personas diversas (Romero, 2007).. Para poder conseguir estos objetivos, las organizaciones españolas deberán usar modelos de gestión que, concilien la diversidad cultural con la cohesión social, permitiendo a las minorías tener las mismas oportunidades que los grupos mayoritarios, valorizando las diferencias y su esencia, evitando la creación de grupos en el empleo y favoreciendo la integración de todos los trabajadores.

La gestión de la diversidad en España se debe considerar como un proceso intercultural que conduce a una adaptación en doble sentido, que promueve la participación y el compromiso de toso los individuos, los cuales interactúan entre ellos, más allá del mero reconocimiento y coexistencia, formando una cultura común, de tolerancia e diversidad (Bauböck, 2004).

Tras hacer estas observaciones concluimos diciendo que a pesar del interés que ha suscitado a nivel gubernamental, la gestión de la diversidad, la literatura sobre esta temática en el mercado laboral español, sigue siendo escasa por esta motivación centraremos nuestro análisis empírico en la recopilación de la información primaria que nos permita aportar más datos en este campo y sus implicaciones con la dirección de empresas.

Como hemos visto anteriormente los estudios sobre la gestión de la diversidad surgieron en Estados Unidos entre los años ' 80 y ' 90 , un país que hoy en día considera la «diversidad cultural» una estrategia para conquistar los mercados. A partir de los años noventa y en esta última década, este asunto, junto con la lucha contra la discriminación, ha empezado a tener un interés creciente incluso en las organizaciones Europeas.

En el ámbito de la gestión de Recursos Humanos, la cuestión de la diversidad entre los trabajadores tiene una especial transcendencia al tratarse de una temática muy presente en las empresas globales. Para saber gestionar y enfrentarse a estos desafíos es necesario reforzar las competencias de los directivos a crear un ambiente inclusivo y de respeto además de un cambio en la cultura organizacional.

La dirección de la diversidad es particularmente apreciada y estudiada por parte de los profesionales en recursos humanos, en los países anglosajones, en España y en otros países europeos. Sin embargo, este tema sigue siendo escasamente conocido y parece haber llegado sólo a un número limitado de empresas (en su mayoría multinacionales).

La falta de claridad acerca de lo que significa y lo que implica "gestionar la diversidad en la empresa», el escepticismo hacia el cambio, que caracteriza a las empresas y la renuncia a invertir cuando las perspectivas de rendimientos tienden a aparecer en el medio y largo plazo y al mismo tiempo, son difíciles de medir y la falta de una «teoría universal» a seguir 
y aplicar, se encuentran entre las principales causas de esta «falta de atención» al tema, que en cambio tiene una fuerte relevancia en términos de organización, económico y social.

La gestión de la diversidad tiene que ser una política de las empresas de todos los tamańos y sectores, y un reto estratégico para su presente y futuro, que debe ser percibido para poder enfrentarse a él.

\section{Bibliografía}

Agocs, Carol; Burr, Catherine. Employment equity, affirmative action and managing diversity: assessing the differences. International Journal of manpower, 1996, vol. 17, no 4/5, p. 30-45.

Alonso, Miguel Pajares. Discriminación racial y políticas antidiscriminatorias. En La discriminación racial: propuestas para una legislación antidiscriminatoria en España. Icaria, 2003. p. 13-38.

Alvarez, Abel F. Losada. The Cuban Labor Market and Immigration from Spain, 1900-1930. Cuban Studies, 1995, p. 147-164

Barberá Heredia, Ester. Diversidad de género, igualdad de oportunidades y entornos laborales. CIRIEC-España, revista de economía pública, social y cooperativa, 2004, no 50.

Bass, Bernard M.; Burger, Philip. Assessment of managers: An international comparison. Free Pr, 1979.

Bassett-Jones, Nigel. The paradox of diversity management, creativity and innovation. Creativity and innovation management, 2005, vol. 14, no 2, p. 169-175.

Bauböck, Rainer. Civic Citizenship-A New Concept for the New Europe. Managing Integration: European Union Responsibilities towards Immigrants, Bertelsmann Foundation, Brussels, 2004, p. 146-63.

Bernet, J. M. D. M. (1994). La inmigración española en Cuba, 1880-1953. In Cuba, la perla de las Antillas. Actas de las I Jornadas sobre» Cuba y su Historia" (pp. 121-136). Ediciones Doce Calles.

Cahn, Steven M. The affirmative action debate. Routledge, 2013.

Charles, Joann. Diversity management: An exploratory assessment of minority group representation in state government. Public Personnel Management, 2003, vol. 32, no 4, p. 561-577.

Child, John. Culture, contingency and capitalism in the cross-national study of organizations. University of Aston Management Centre, 1979.

Chinchilla-Albiol, María Nuria; Cruz-Rivas, Hugo Donaldo. Diversidad y paradigmas de empresa: un nuevo enfoque. 2011.

Coate, Stephen; Loury, Glenn C. Will affirmative-action policies eliminate negative stereotypes?. The American Economic Review, 1993, p. 1220-1240.

Cockburn, Cynthia. Equal opportunities: the short and long agenda. Industrial Relations Journal, 1989, vol. 20, no 3, p. 213-225. 
Comision Europea (2008): "Continuing the Diversity Journey: Business Practices, Perspectives and Benefits». Office for Official Publications of the European Communities, Luxembourg.

Ezorsky, Gertrude. Racism and justice: The case for affirmative action. Cornell University Press, 1991.

Fambuena, Aurora; Esquiza, Carmiña Moya. Movimientos migratorios hoy: Aproximación a la Sociología de las migraciones. Nau llibres, 1997.

Fine, Marlene G. Cultural diversity in the workplace: The state of the field. Journal of Business Communication, 1996, vol. 33, no 4, p. 485-502.

Garrison, William A.; Modigliani, Andre. The changing culture of affirmative action. Equal employment opportunity: labor market discrimination and public policy, 1994, vol. 373.

Gibbon, Peter. Equal opportunities policy and race equality. Critical Social Policy, 1990 , vol. 10 , no 28 , p. $5-24$

Gilbert, Jacqueline A.; Stead, Bette Ann; Ivancevich, John M. Diversity management: A new organizational paradigm. Journal of business ethics, 1999, vol. 21, no 1, p. 61-76.

González, Santiago Sánchez. La lucha contra la desigualdad: Acciones positivas y derechos socioeconómicos en Estados Unidos y en la India. Revista Derecho Público Iberoamericano, 2014, no 4, p. 65-99.

Gozálvez Pérez, Vicente. Los extranjeros residentes en España: su aportación a la demografía. 2010.

Hernández, Antonio Manuel Macías. Un siglo de emigración canaria: 1830-1930. En Españoles hacia América: la emigración en masa, 1880-1930. Alianza Editorial, 1995. p. 166-204.

Hernández, Ricardo Robledo. Crisis agraria y éxodo rural: emigración española a ultramar, 1880-1920. En La crisis agraria de finales del siglo XIX:(I Seminari Internacional d'historia de Girona). Crítica, 1988. p. 212-244.

Herring, Cedric; Henderson, Loren. From affirmative action to diversity: Toward a critical diversity perspective. Critical Sociology, 2012, vol. 38, no 5, p. 629-643.

Hofstede, Geert. Culture and organizations. International Studies of Management \& Organization, 1980, vol. 10, no 4, p. 15-41.

Holzer, Harry; Neumark, David. Assessing affirmative action. National bureau of economic research, 1999.

INE (Instituto Nacional de Estadística) 2015: «Extranjeros residentes en Espańa, estadísticas del Padrón Municipal». Cifras INE.

Janssens, Maddy; Steyaert, Chris. Theories of diversity within organisation studies: Debates and future trajectories. 2003.

Kalev, Alexandra; Dobbin, Frank; Kelly, Erin. Best practices or best guesses? Assessing the efficacy of corporate affirmative action and diversity policies. American sociological review, 2006, vol. 71, no 4, p. 589-617.

Kelly, Erin; Dobbin, Frank. How affirmative action became diversity management employer response to antidiscrimination law, 1961 to 1996. American Behavioral Scientist, 1998, vol. 41, no 7, p. 960-984. 
Kersten, Astrid. Diversity management: Dialogue, dialectics and diversion. Journal of Organizational Change Management, 2000, vol. 13, no 3, p. 235-248.

Kravitz, David A. The diversity-validity dilemma: Beyond selection - the role of affirmative action. Personnel Psychology, 2008, vol. 61, no 1, p. 173-193.

Lafuente, Gloria Sanz. Emigrantes industriosos: los trabajadores españoles y el «milagro económico alemán», 1960-1985. Revista de Historia Industrial, 2015, no 60, p. 173-216.

Lahey, Joanna N. Do older workers face discrimination?. Center for Retirement Research at Boston College, 2005.

Lempert, Richard. Affirmative Action in the United States: A Brief Summary of the Law and Social Science. U of Michigan Public Law Research Paper, 2014, no 430.

Leonard, Jonathan S. The impact of affirmative action on employment. 1984.

Liff, S. Equal opportunities: continuing discrimination in a context of formal equality. Industrial Relations in Britain, Blackwell, Oxford, 1995.

Naranjo Orovio, C., \& Santamaría García, A. (2000). De España a las Antillas. Historia e historiografía de la migración española a Puerto Rico y Cuba en las últimas décadas del siglo XIX y primeras del XX.

Ortlieb, Renate; Sieben, Barbara. Diversity strategies and business logic: why do companies employ ethnic minorities?. Group \& Organization Management, 2013, p. 1059601113497094.

Osuna, Jacinto Rodríguez. Migraciones exteriores, transición demográfica y proceso de desarrollo. Reis, 1985, no 32, p. 27-42.

Palazón Ferrando, S.: «La emigración española a latinoamérica (1946-1990). Reanudación y crisis de un flujo secular».

Estudios Geográficos , LIV, 210, 1993

Pfeffer, Jeffrey. Some consequences of organizational demography: Potential impacts of an aging work force on formal organizations. Graduate School of Business, Stanford University, 1979.

Podsiadlowski, Astrid, et al. Managing a culturally diverse workforce: Diversity perspectives in organizations. International Journal of Intercultural Relations, 2013, vol. 37, no 2, p. 159-175.

Ramos, R.; Sanromá, E.; Simón, H. Diferencias salariales entre sector público y privado según tipo de contrato. mimeo, 2013.

Romero, Francisco Sacristán. Evolución de las políticas activas de empleo para inmigrantes en España. Revista de História Regional, 2007, vol. 10, no 2.

Ronda-Pérez, Elena, et al. Condiciones de trabajo y salud de los trabajadores inmigrantes en España: revisión bibliográfica. Revista Española de Salud Pública, 2014, vol. 88, no 6, p. 703-714.

Rutherglen, George A. The Origins of Arguments Over Reverse Discrimination: Lessons from the Civil Rights Act of 1866. Virginia Public Law and Legal Theory Research Paper, 2014, no 2014-14.

Salazar, Gloria González. Mercado de Trabajo e Integracion Social. Investigación Económica, 1974, vol. 33, no 130, p. 231-255. 
Sanz Lafuente, Gloria. Industrial emigrants: Spanish workforce and the German"economic miracle", 1960-1985. Revista de Historia Industrial, 2015, no 60, p. 173-216.

Sartori, Giovanni. Elementos de teoría política», alianza editorial. 1992.

Scheepers, Sarah. Discourses on diversity in the Belgian and Flemish public sector. 2007.

Skrentny, John D. Inventing race. Public Interest, 2002, no 146, p. 97.

Slater, Stanley F.; Weigand, Robert A.; Zwirlein, Thomas J. The business case for commitment to diversity. Business horizons, 2008, vol. 51, no 3, p. 201-209.

Steger, Mark; Erwee, R. Managing diversity in the public sector: A case study of a small city council. International Journal of Organisational Behaviour, 2001, vol. 4, no 1, p. 77-95.

Stone, Katherine VW; Arthurs, Harry (ed.). Rethinking workplace regulation: Beyond the standard contract of employment. Russell Sage Foundation, 2013.

Tawney, Richard Henry. The acquisitive society. Courier Corporation, 1961.

Thomas JR, R. Roosevelt. Managing diversity: A conceptual framework. 1992.

Uri, Noel D.; Mixon JR, J. Wilson. Effects of US equal employment opportunity and affirmative action programs on women's employment stability. Quality and Quantity, 1992, vol. 26, no 2, p. 113-126.

Wejnert, Barbara. Reflections on the impact of globalization: Diffusion, equality, and opportunities. Voices of Globalization (Research in Political Sociology, Volume 21) Emerald Group Publishing Limited, 2014, vol. 21, p. 255-261.

Yang, Yang; Konrad, Alison M. Understanding diversity management practices: Implications of institutional theory and resource-based theory. Group \& Organization Management, 2011, vol. 36, no 1, p. 6-38.

Zapata-Barrero, Ricard. Managing diversity in Spanish society: a practical approach. Journal of Intercultural Studies, 2010, vol. 31, no 4, p. 383-402.

Ziliani, Estefanía. La acción afirmativa en el derecho norteamericano. Revista Electrónica del Instituto de Investigaciones «Ambrosio L. Gioja, 2011, vol. 5. 